\title{
Exploration and Analysis of the Application of Chinese Etymology in the Chinese Character Font Design
}

\author{
Ting Yao \\ Eastern International Art College \\ Zhengzhou University of Light Industry \\ Zhengzhou, China
}

\begin{abstract}
According to the Chinese etymology, the Chinese character font design is based on the study on information and the origin of Chinese characters in each historical period of the concrete structure form. And under the premise of retaining the original information of Chinese characters, we manage to design Chinese characters. Such as oracle, Chinese bronze inscription and seal character, these three glyphs is reorganized and designed in the hand of the book Shuo Wen Jie $\mathrm{Zi}$ (Chinese first official dictionary). The idea of design not only is helpful to know the first origin of Chinese characters change and the meaning of the word in each historical period, also benefit to learn the better form of Chinese characters besides simplified Chinese and traditional Chinese. It's unfair that people take an indifferent attitude to it and then decide it just history and recorded. I think it is necessary to explore and future design the Chinese characters. And it must show the charm of our national characters after contemporary design in the whole new look.
\end{abstract}

Keywords-Chinese etymology; font structure; glyph of aesthetic

\section{EXPLORATION AND ANALYSIS OF THE AESTHETIC OF ANCIENT CHINESE CHARACTERS}

The origin of Chinese character can be traced back to the mythical Cang Jie who creates new characters, and also to the primitive period when people cannot communicate with each other in basic language. Whether in any historical period, Chinese characters exist, evolve, and are used in various forms. But in general, the formation of Chinese characters follows the six methods making words, namely Liu Shu (six categories of Chinese characters). Liu Shu summarizes the Chinese characters form and divides them into: pictograph, self-explanatory, ideograph, associative compound, transcription, borrowing, which are explained in details in the book Shuowenjiezi by $\mathrm{Xu}$ Shen from Dong Han dynasty: According to rituals in the Zhou Dynasty, children in the scholar family should go to school at 8 years old and teachers should take Liushu as textbook. The first category refers to self-explanatory characters which means you can recognize the shape and structure of characters but if you want to know what the characters mean you must learn and study on them such as Chinese characters “上” and
“下”. The second category refers to photography which means you should depict the characters in drawing method in accordance with the corresponding gestures and shapes of the described objects such as Chinese characters “日” and “月”. The third category refers to associative compound which means you can choose the neighboring sound symbols and meaning symbols to collaborate words according to the natures and names of described objects such as “江” and “河”. The fourth category refers to ideograph which means a sign or symbol that represents a particular idea or thing rather than a word such as Chinese characters “武” and “信”. The fifth category refers to transcription which means setting a character as an affix and a root to create new characters and the pronunciation and meaning of the new characters are partly similar to the affix and root such as Chinese characters “考” and “老”. The last one is borrowing which means there is no derivation for a character and you just find a homophone to represent the described object such as Chinese characters “令” and “长”.

Firstly, there are six methods in the word-formation means that the formation of Chinese characters is ever changing. but the ever-changing is not only showed in the simplified Chinese, also including oracle, Chinese bronze inscription and seal character(minor and seal script), clerical script and so on, and it can improves the creation possibility of designing the future Chinese character. In addition, the existence and evolution of Chinese character has a long history. Lateral perspective from history, different countries and areas has the different writings of the Chinese character during the same period. And the Chinese character also will change because of its original information elements under the influence of human beings, some words will increase or decrease in the composition, some words will have great changes in the formations. But because of these, it is beneficial to improve more refer to elements and basics for the design of Chinese character. From the analysis of the detailed, the difference in the Chinese character component radical, overall construction and information elements (including the source of original elements, graphic design, etc.) provided the infinite possibility for the future design of Chinese characters font. 


\section{SOME SimPLE EXAMPLES OF ANALYZING THE AEsTHETIC OF ANCIENT CHINESE CHARACTERS.}

明(Ming): “明(Ming)” is a knowing word. In oracle, the sun and the moon will shine brightly, so they are the symbols of brightness. The evolution of “明(Ming)” from oracle to Chinese bronze inscription to regular script can be seen in Fig. 1:

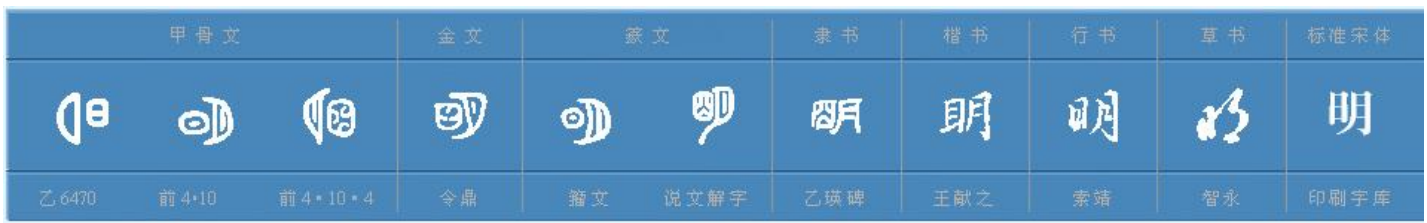

Fig. 1.

“明(Ming)” is the hieroglyphics of oracle “日”and “月”. From oracle to seal letter, “明(Ming)” is more inclined to be showed in graphic form, which is figurative and the original graphics information is clear.

Although simplified Chinese is the most easy to be identified and used in the Chinese characters evolution, but because of this evolution, doing a design in the font itself or on the simplified words will be vulnerable to limit, and it's difficult to break through the design of general category.
Ancient Chinese character is diverse in the stroke structure, bending and straight, and is more aesthetic than simplified Chinese.

\section{B. Exploration and Analysis of the Character “胃(Wei)”}

胃 (Wei): From seal script font, you can see that “胃 (Wei)"is consisted of a bag with rice in the top and the meat in the bottom, meaning that it is an organ which people use to storage and digest food. The font evolution can be seen in Fig. 2:

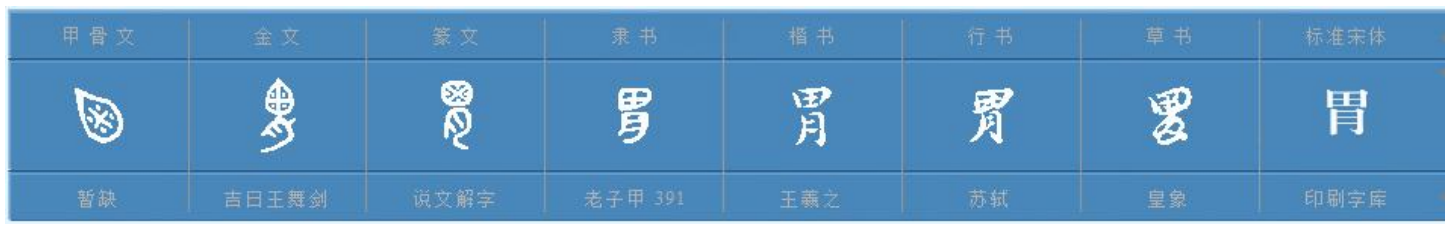

Fig. 2.

“胃 (Wei)” is composed of upper and lower structure hieroglyphics, and during the period of the original oracle evolving into the seal script, there are some increase and decrease changes in the character font, and some changes in the form. Even the visual communication of seal script is much better in the integral beautification. Oblique cross and dot in the top part of the word “胃(Wei)” does not appear the stroke writing of simplified Chinese. To some extent, it tends to reflect dot, line and surface in the planar formation. It not only contains abundant text messages, also possesses the aesthetic of formation. These beautiful letter symbols is gradually forgotten by the human beings, and isn't it worthwhile for people to learn and remember it.

\section{Exploration and Analysis of the Character “周 (Zhou) ",}

周 (Zhou) :Seen from the oracle glyph, there are four points in the inside of “周 (Zhou)", which means close. However the master of seal script, Duan Yucai, thinks that the character “周 (Zhou)" comes from using the mouth, and what is good at the mouth is close. The evolution of character sources can be seen in Fig.3:

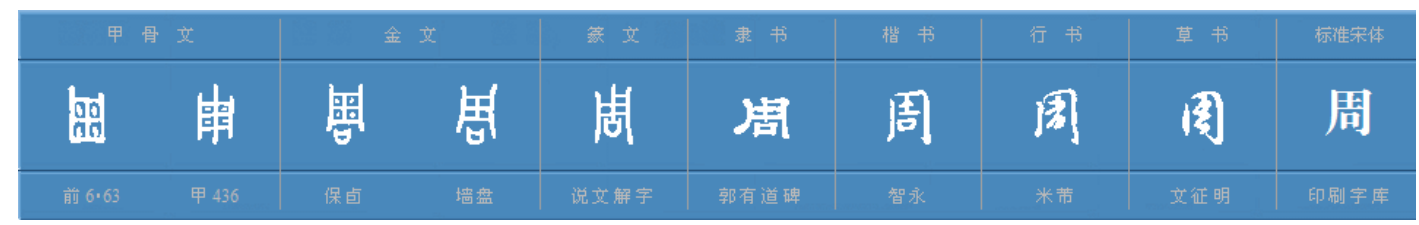

Fig. 3.

The evolution of the character “周(zhou)" looks like a process of constant improvement. But the meaning of the original character formations is different, and “周(zhou)" in the oracle and in the seal script has different origin explanations. Considering the aesthetics, individuals are more like Chinese bronze inscription " 周 (zhou)". In massive structure, the Chinese bronze inscription "周 (zhou)" is different from simplified Chinese "周(zhou)",light and heavy, 
thin and dense, and does not have uniform structures like simplified Chinese. Chinese bronze inscription "周(zhou)" is more like a symbol with the complete composition, also like a separate logo. A variety of written forms can be adjusted locally, and there is stronger plasticity in the viewpoint of designs.

From the above cases, we can see that Chinese character has rich changes in the Oracle, Chinese bronze inscription and seal script. Whether in structure, local graphic image or text, the information contained in those is extremely rich and has a profound culture. For the people always using the simplified Chinese, it not only has an effect on our visions, but also supports the source of Chinese characters for us. And we can have a profound analysis of our native characters and read it. That will provide the new designed look of Chinese characters with sufficient basic and creative possibilities.

\section{THE DESIGN DEPENDING ON THE INFORMATION ELEMENTS OF ANCIENT CHARACTERS}

Whether individual characters or combined characters, there glyph elements, like Chinese character component radicals and image information contained in the word itself. But if it exceed the limit of design, making the original character unrecognized or deviating from the meaning of the word itself, then the design will lost the meaning of itself. So it is the purpose and meaning of design to master the limit and design the font elegant. Since the aesthetic elements of Chinese characters are rich and colorful, naturally, a variety of design points and design methods will exist.

\section{A. Study and Design of the Character “土 (Tu) ” 土 ( Tu ): As shown in Fig.4}

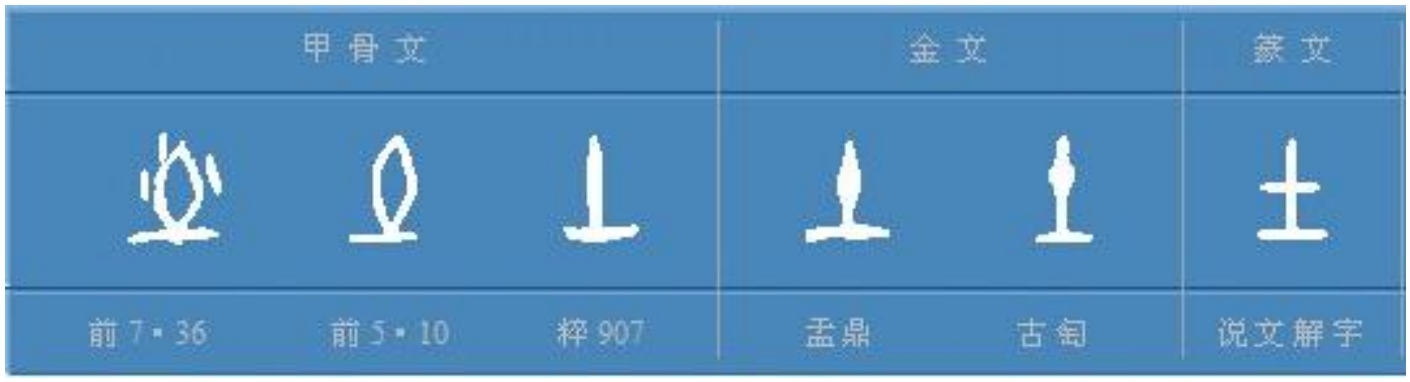

Fig. 4.

Firstly, we should learn about the original meaning of “土 ( $\mathrm{Tu}$ ) ", which means the mound on the horizon. Three points on the mound in oracle means splashed dust. It more vividly describes the original meaning of “土（Tu）”. Despite the structure of “土 ( $\mathrm{Tu}$ ) " is very simple, but in combination with oracle and bronze inscription, the present"

( Tu ) " can be designed. Shown in Fig.5:

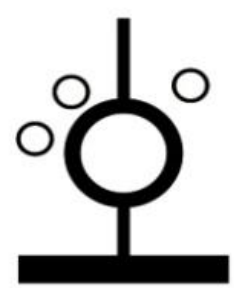

In the bronze inscription form of “土 ( $\mathrm{Tu}$ )",the solid circle is designed into a big scale circle, referred a mound, and the splashed dust is designed into some little circle attached the big circle. The design does not damage the inherent structure of the font, but make the word"土（Tu）" have a new look, and it also abandons the traditional cross and vertical in the simplified Chinese. With the circle element in the composition, the word"土（Tu）" is more powerful , and more beautiful in the form.

\section{B. Study and Design of the Character “束 (Shu) ” 束 (Shu) As shown in the Fig. 6}

Fig. 5. 


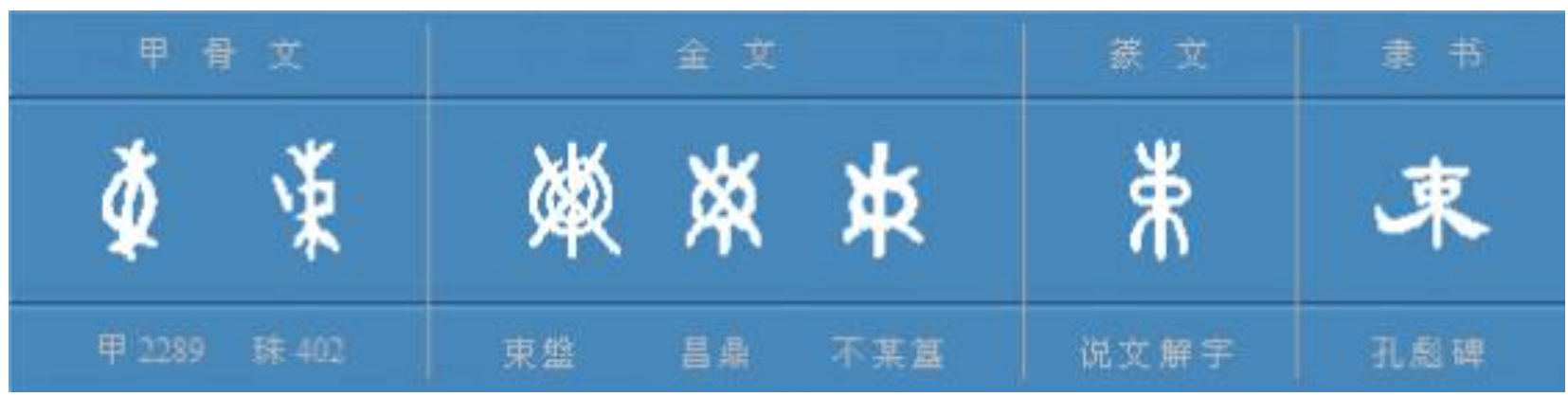

Fig. 6 .

The create meaning of “束 (Shu) ” indicates the luggage bundled books, but in the bronze inscription, it specially indicates the knot binding up the luggage. Now that we know the original create meaning of the character, and there are graphics expressing binding knots in the bronze inscription. We can refer to the bronze inscription “束 (Shu) "and seal letter “束 (Shu) ” and do a combining design to create a new font of “束 (Shu) ”. As shown in Fig. 7

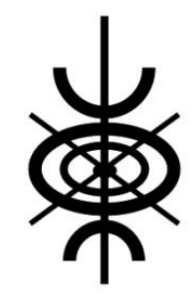

Fig. 7.

The design of “束（Shu）"is different from the common Chinese characters with all the elements combined and ruled in modern methods.

It has a strong symmetric structure combined bronze inscription type and seal script type, and the combination use a oval represent a cord, add a knot in the center. The character
“束 (Shu) ” show a modern and simple look, with the oblique cross in the bronze inscription still reserved. These characters with double attributes text and graphic symbol, is the result of combining ancient characters and modern design methods.

\section{IV. THE NEW CREATED STRUCTURE DEPENDING ON THE ANALYSIS OF ANCIENT CHINESE CHARACTERS}

The font structure of simplified Chinese is divided into up and down type, left and right type, half a pack type, package type and mosaic. However, under the premise of the basic and rich information elements existing in the ancient character, it is possible to create some new structure types. These characters will show their new look, such as overlay, overlapping, palace, and perspective types, etc. In the development of Chinese characters, few people will use this breakthrough point to design the character. Now that the ancient Chinese character meets such condition, so considering another new method, let us appreciate the charm of the brand new Chinese characters.

\section{A. Study and Design of the Overlay Structure “晶 (Jing) ”}

The overlay type: 晶 ( Jing), as shown in Fig. 8

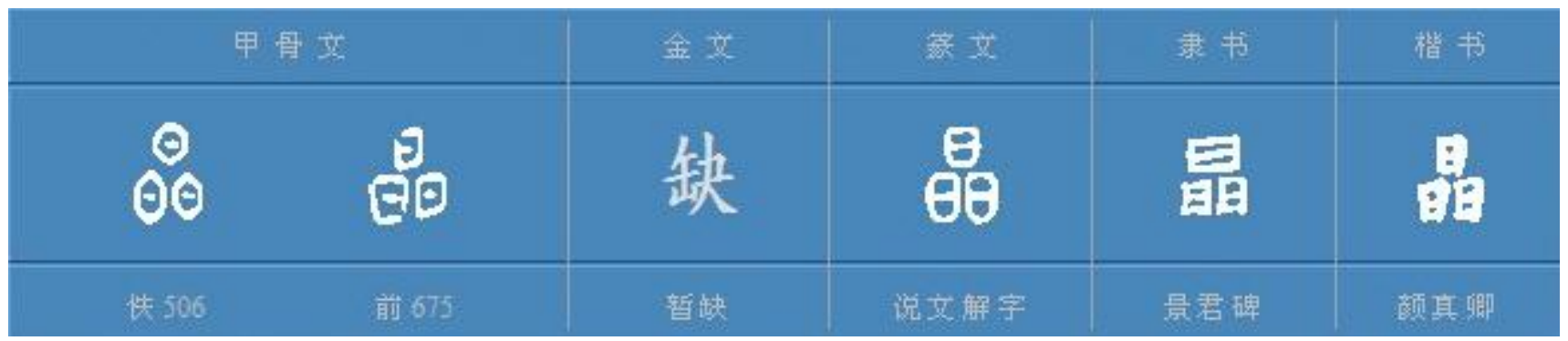

Fig. 8.

“晶（Jing）” is composed of three “日(Ri)”. “日(Ri)” on the behalf of the sun, is a shining celestial body. So the meaning of the character “晶 (Jing)" is the symbol of multiple light constellation. However, the character “晶
(Jing)" is linked with the crystal, and its first impression is transparent, just like a regular crystal. As the constitutive structure of crystal, the writing style of “晶（Jing）” always 
consists of three characters “日(Ri)". So it is designed like Fig.9.

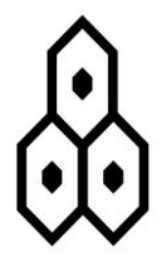

Fig. 9.
Strictly speaking, the combining form of “晶（Jing）” is a type of up and down. Now design each unite "日(Ri)" into the crystal structure form of a regular hexagon, and make it overlapped in the liked form of crystal molecules to create the new character “晶 (Jing) ”. But the font structure is almost non-existent in Chinese characters.

\section{B. Study and Design of the Overlapped Structure “由 (You) "}

The overlapped type: “由（You）”, as shown in Fig. 10

\begin{tabular}{|c|c|c|c|c|c|}
\hline 甲 肖 文 & 金 & & 立 & 逮 书 & 指 书 \\
\hline 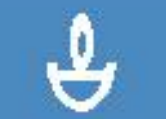 & & & 불 & $1 \div$ & \\
\hline 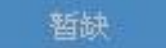 & 整缺 & 復站 & 说䒵解宇 & 挑 & 点世南 \\
\hline
\end{tabular}

Fig. 10.

The character “由（You）”is the same as “油（You）”, and the top in oracle and bronze inscription signify oil drop, the bottom signify a vessel. so the original intention of making the word "由 (You)" is to put oil drop into the mouth of a vessel. The source information of character is associated with the condition of dripping water, and in the simplified character “由 (You)", the vertical on behalf of oil drop is already dripped into the vessel. So according to the oracle and bronze inscription, the “由（You）"can be designed as Fig.11.
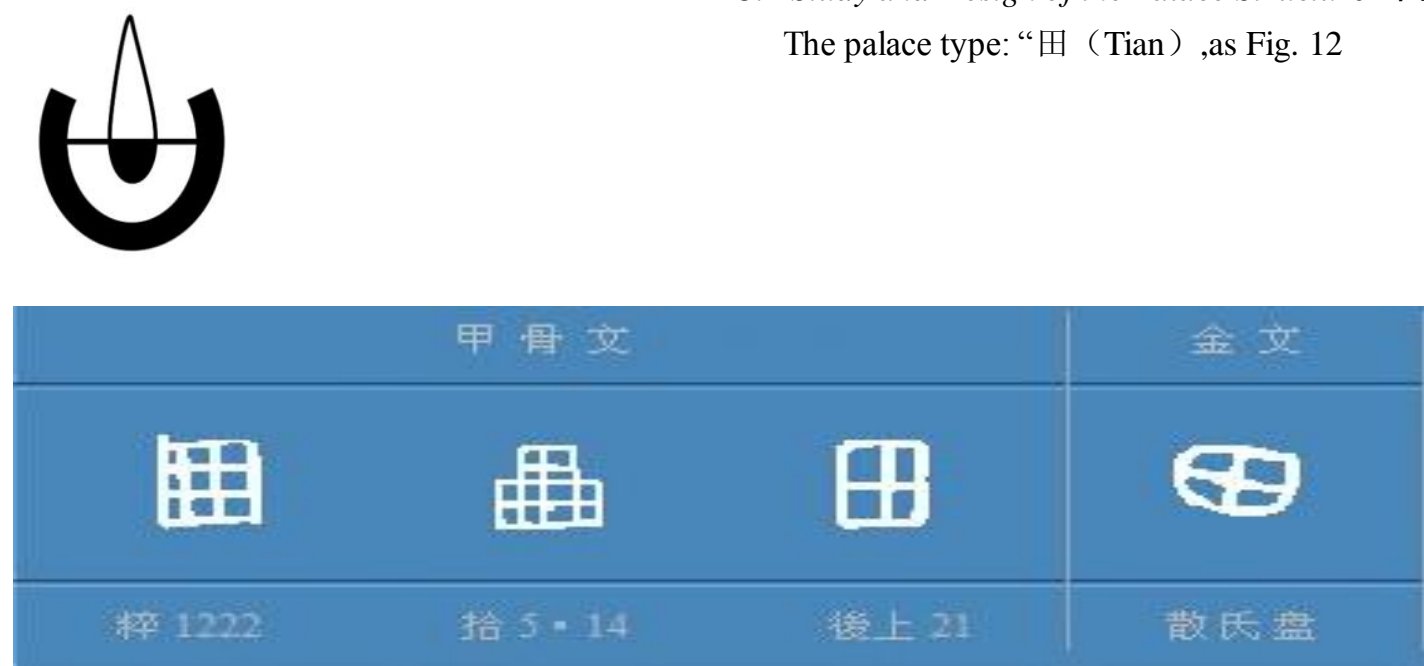

Fig. 12.

The intention of making the word “田（Tian）"is to show the crisscross footbath in a cloud of field ridges. One method
Fig. 11.

This overlapped design makes the explanation of the oracle “由 (You) "more vivid. The black in the bottom indicates that an oil drop has been dripped into the vessel. It's just like the condition that "a vertical line" comes into the mouth of the vessel in the writing of simplified Chinese. And the oil drop represents a more figurative graph, a single glyph with the stronger visual communication.

C. Study and Design of the Palace Structure “田 (Tian) ” The palace type: “田（Tian）,as Fig. 12 
in the oracle writing is unique, but after being adjusted and planned, it will show another different visual effect. As shown in Fig. 13.

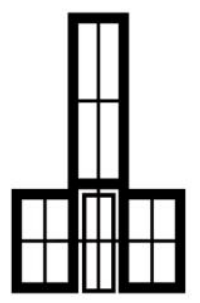

Fig. 13.
The new look of “田（Tian）" is designed on the basic of a writing method in oracle. With the structural composition integrated and the overall proportion allocated and adjusted, the palace type of the character “田 (Tian)" is designed. However ,due to the character in palace type is extremely rare, the word"田（Tian）"created in the font design method looks like more unique and beautiful. This font combination is unprecedented, so the way of designing the new character look may be a bit exaggerated. But the results are not very contradictory, so this design should be reasonable.

\section{Study and Design of the Perspective Structure “门 (Men) "}

The perspective type: “门 (Men) ”as shown in Fig. 14

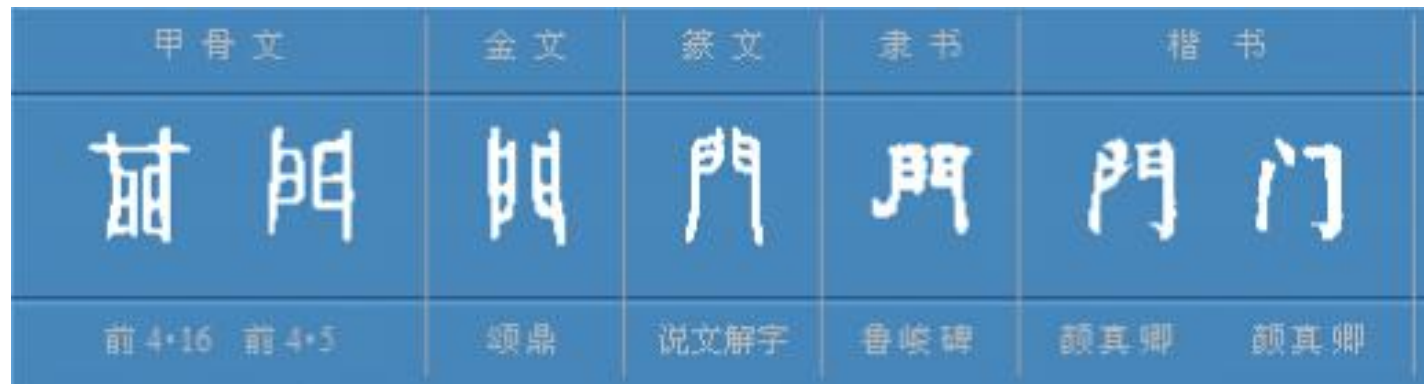

Fig. 14.

The intention of making the character "门 (Men) "in oracle is that: the top part likes “\#” indicates an entrance to the house, and the bottom part is a double doors, so "门

( Men ) " is a pictographic character. The symmetrical character like this designed will show a perspective effect.

As shown in Fig. 15:

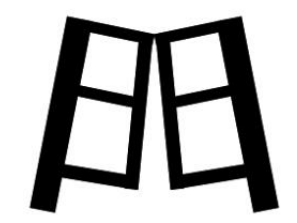

Fig. 15.

Such the character “门 (Men)" not only reserves the former structural type and appears lively because of the perspective, but also freeze-frames the condition of opening the door. It is also easy to be identified and put into use with interests. And because its method and angle of design are flexible, the created character won't be monotonous and deviate from the original meaning or lose it. It is the unexpected sense to endow a new look of the character and open up a new idea of designing Chinese characters fonts. So the design is valuable and significant.

Even though the above example is just picking up some Chinese characters randomly and coming into design, I hope the designed method can draw forth much better ideas. The method is enough to prove that the beauty of Chinese characters is absolute, and its existence will support the creative geography.

Through using the origin of Chinese character to design the brand -new character, it will be an evolution about the design method of Chinese character, and it will have a far- reaching significance. With the gradual indifference to the culture of Chinese characters, we should allocate the design of Chinese character. On the one hand, it can help more people understand and have a thorough read about our Chinese characters, know the importance of our national artistic cultural treasures and understand the great and profound significance of our Chinese character culture. On the other hand, as shown from the aesthetic perspective, it not just breaks the old designed angle of Chinese character, also dares to put forward new methods of structure. In addition, a new Chinese character contains the original message and enhances the aesthetic to make it more beautiful, which caters to the trend to the present aesthetic. This is a bold idea, is also a bold practice. I hope the pattern can affect more people, and inspire people to learn about the culture of Chinese characters. And I also hope that our country don't make the once so beautiful Chinese character as a history. 
It should be popped up slowly, and it will be an eternal shining golden light in the world's words.

\section{REFERENCES}

[1] Xu Shen.Shuo Wen Jie Zi [M].Beijing: Zhonghua Book Company Press.2001

[2] Zhang Daoyi. The Beautiful Chinese Characters [M].Shanghai Literature and Art Publishing House.2003

[3] Lv Shengzhong. Characters of Artistic Conception [M], China Youth Publishing House. 2000

[4] Tong Manzhi. Hundred Types of Modern Artistic Calligraphy [M], Changsha: Hunan Fine Arts Publishing House, 1991.

[5] Wang Chenglong, Tong Manzhi. Calligraphy and Artistic Calligraphy [M], Changsha: Hunan Fine Arts Publishing House, 1993.

[6] Wei Jinan, Li Qiang.The Practical Artistic Calligraphy [M] Beijing: Knowledge Press, 1991.

[7] Wen Yibo. One Thousand Kind of New Artistic Calligraphy [J], Hong Kong: Xintu Art Publishing House, 1978.

[8] Yu Bingnan. Artistic Calligraphy [M], Beijing: People's Fine Arts Publishing House, 1980.

[9] Zhang Zhilin. Decorative Artistic Calligraphy [M], Shanghai: Shanghai People's Fine Arts Publishing House, 1983.

[10] Zhang Zhilin. Practical Decorative Artistic Calligraphy [M], Beijing: Hope press, 1988.

[11] Guo Cunshan. Artistic Calligraphy of Frequently-used Words of Business [M]. Beijing: China Pictorial Publishing House, 1996.

[12] Wen Yunrong. Variant of Advertising Artistic Calligraphy and Logo Design [J], Beijing: Minzu University of China Press, 1994.

[13] Yu Nengzhi. Frequently-used Variant Artistic Calligraphy [M]. Beijing: China Pictorial Publishing House, 1999.

[14] Wang Xinzhu. Hundred Types of Artistic Calligraphy [M], Beijing: Shield Press, 2000.

[15] Jin Xianghong. Design of Modern Commercial Artistic Font [M], Nanjing: Jiangsu Science and Technology Press, 2001. 